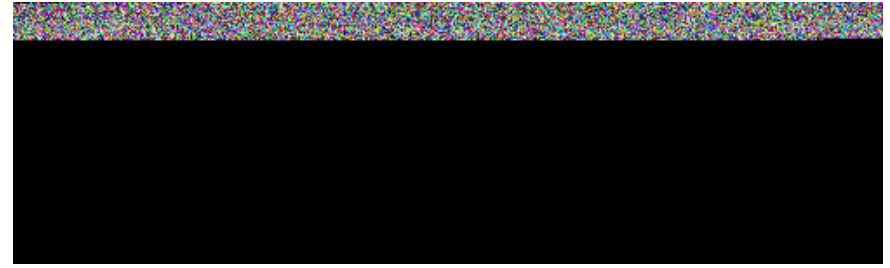

This information is current as of April 26, 2023.

\title{
Zuckerkandl Tubercle of the Thyroid: A Common Imaging Finding That May Mimic Pathology
}

T.C. Lee, S.K. Selvarajan, H. Curtin and S. Mukundan

AJNR Am J Neuroradiol 2012, 33 (6) 1134-1138

doi: https://doi.org/10.3174/ajnr.A2914

http://www.ajnr.org/content/33/6/1134 


\section{ORIGINAL RESEARCH}

T.C. Lee

S.K. Selvarajan

H. Curtin

S. Mukundan

\title{
Zuckerkandl Tubercle of the Thyroid: A Common Imaging Finding That May Mimic Pathology
}

\begin{abstract}
BACKGROUND AND PURPOSE: The posterior thyroid tubercle, also known as ZT, is an important surgical landmark due to its close proximity to the recurrent laryngeal nerve. A recent case of ZT with a nodular configuration caused clinical concern but was shown on biopsy to be normal thyroid tissue. The purpose of this study was to review a series of CT neck studies to identify how often ZTspecifically, a nodular subtype-was present.
\end{abstract}

MATERIALS AND METHODS: A total of 96 neck CTs from sequential patients were retrospectively identified from January 2010 to July 2010. ZT was defined on imaging as a thyroid lobe extending posterior to the tracheoesophageal groove. A nodular subtype was defined as having a narrowed neck at the level of the tracheoesophageal groove.

RESULTS: There were 31 women and 45 men (mean age of 56.1 years, range 22-100 years) who met inclusion criteria. Sixty-seven patients had thyroid glands that extended posterior to the tracheoesophageal groove at CT imaging; this finding was bilateral in 43 patients, more commonly on the right 60 versus 49). Thirty-two of these patients ( $42.1 \% ; 16$ male, 16 female) had a nodular subtype.

CoNCLUSIONS: ZT is an important surgical landmark of the thyroid that has important radiologic variations. It has a nodular shape in over a third of CT neck studies in our series. Recognition of this feature of the thyroid gland at CT imaging can obviate the need for biopsy and avoid potential recurrent laryngeal nerve injury.

ABBREVIATIONS: $\mathrm{ABR}=$ American Board of Radiology; ZT $=$ Zuckerkandl tubercle

$\mathbf{Z}$ $\mathrm{T}$ is a normal protuberance of the thyroid gland arising from the posterolateral margin ${ }^{1}$ (Fig 1). This tubercle has been documented in the surgical literature due to its relevance in locating the recurrent laryngeal nerve. ${ }^{2,3}$ Autopsies in 20 patients showed the ZT to be a useful normal anatomic landmark for identifying the superior parathyroid gland and the recurrent laryngeal nerve. ${ }^{4}$ Although it is a normal landmark in surgery, ZT has radiologic variations. It is not widely recognized in the radiology literature and is sometimes simply described as an indentation on the thyroid lobe caused by the common carotid artery. ${ }^{5}$ Although sonography is the frontline imaging method for evaluating the thyroid gland, the thyroid is imaged on all neck CTs, and recognition of ZT is important, as it can be mistaken for a worrisome mass or metastatic lymph node by an unwary CT interpreter, particularly when streak artifact from the shoulders lowers its attenuation.

Although this tubercle is named after Austrian anatomist Emil Zuckerkandl, who described it in 1902 as processus posterior glandulae thyroideae, ${ }^{6}$ it was first described by the German surgeon Otto Wilhelm Madelung in 1867 as the posterior horn of the thyroid. The thyroid gland develops from 3 anlages: the larger median anlage and the paired smaller lateral anlages. The median anlage arises as an epithelial thickening in the ventral pharyngeal wall, in an area known as the tuberculum impar at the level of the second branchial arch. ZT results

Received April 27, 2011; accepted after revision September 15.

From the Department of Radiology (T.C.L., S.K.S., H.C., S.M.), Brigham \& Women's Hospital, Boston, Massachusetts; and Massachusetts Eye and Ear Infirmary (H.C.), Harvard Medical School, Boston, Massachusetts.

Please address correspondence to Thomas C. Lee, Brigham \& Women's Hospital, Department of Radiology, PBB3, Room 339, 75 Francis St, Boston, MA 02115; e-mail: tclee@ post.harvard.edu

http://dx.doi.org/10.3174/ajnr.A2914

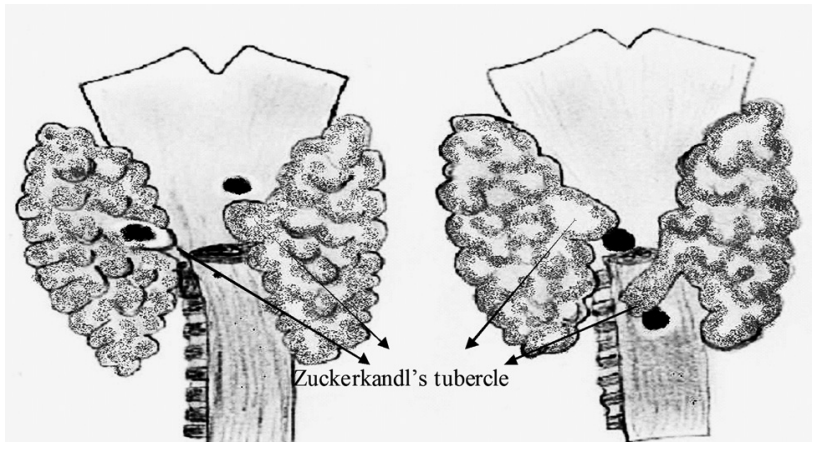

Fig 1. Drawings of 2 different thyroid glands as seen from a posterior point of view, with their relationships to the thyroid cartilage and a cut portion of the esophagus. The arrows indicate ZT and black dots indicate parathyroid glands.

from the embryologic fusion of the lateral thyroid anlages of the fourth pharyngeal pouch and the ultimobranchial body with the median anlage. The ZT is a normal feature in all thyroid glands but is subject to anatomic and radiologic variation. It appears as a thickening where the anlages fuse. When enlarged, it may develop into a nodular process, with the recurrent laryngeal nerve usually passing medial to it in the tracheoesophageal groove. This usual anatomic relation was stressed in studies of thyroidectomy technique that emphasized use of the tubercle in locating the recurrent laryngeal nerve. ${ }^{7}$

A grading system based on the size of the tubercle was proposed by Pelizzo et $\mathrm{al}^{8}$ : grade 0 , unrecognizable; grade 1 , only a thickening of the lateral edge of the thyroid lobe; grade 2, smaller than $1 \mathrm{~cm}$; and grade 3 , larger than $1 \mathrm{~cm}$.

This extension can appear as a nodular mass arising from the posterolateral margin of the thyroid on axial imaging. In contrast, the pyramidal lobe appears as a fingerlike projection pointing in the cranial direction from the isthmus. ZT can also 

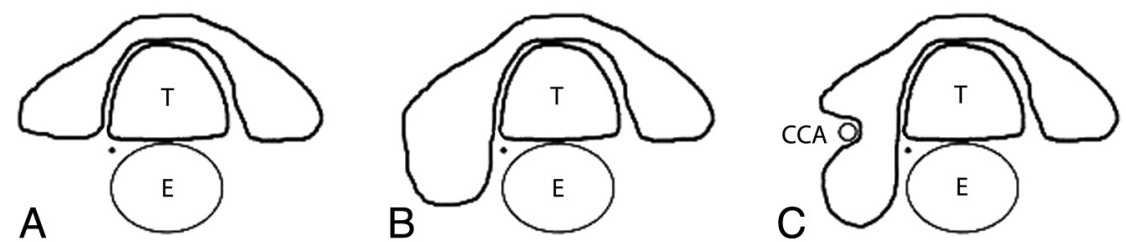

Fig 2. Axial schematic representation of the thyroid ( $\mathrm{T}=$ trachea, $\mathrm{E}=$ esophagus, black dot represents the right recurrent laryngeal nerve). $A$, Thyroid without apparent $\mathrm{ZT}$. $B$, Thyroid with apparent right ZT. C, Thyroid with nodular right ZT (CCA = right common carotid artery within a lateral indentation of the thyroid contour).
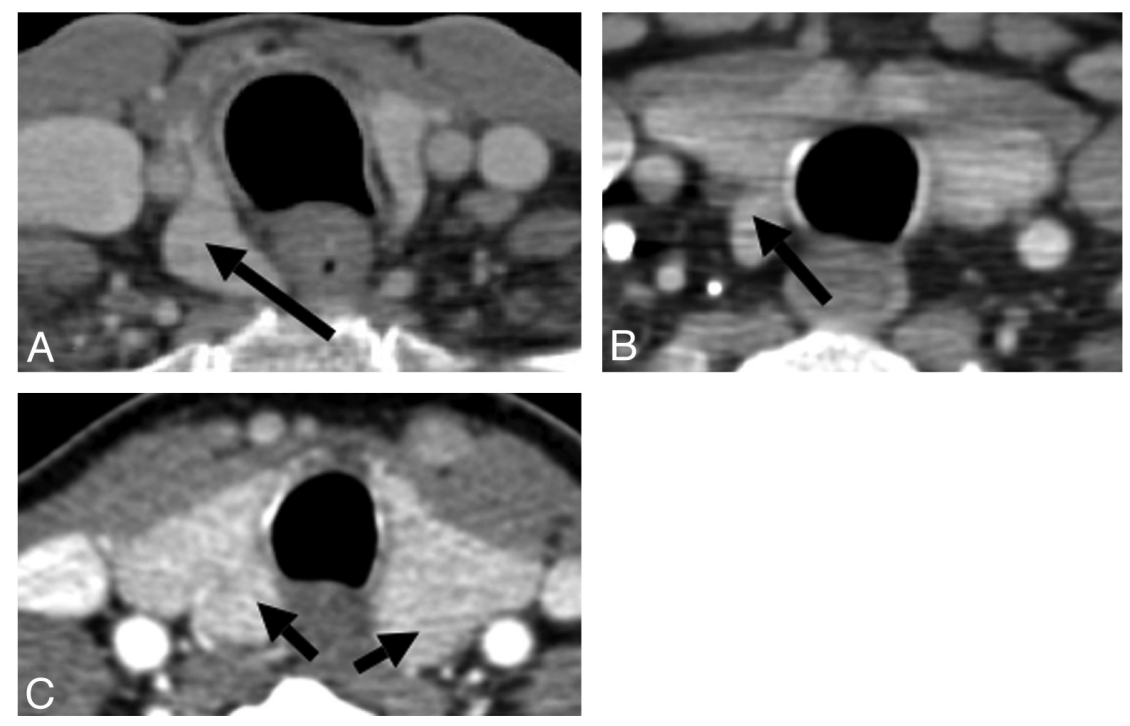

Fig 3. Axial CT scan images demonstrate 3 different appearances of the ZT (arrows).

\begin{tabular}{lc}
\hline $\begin{array}{l}\text { Number of CTs with demographic profile and distribution of ZT in } \\
\text { our review }\end{array}$ & $n$ \\
\hline & 96 \\
\hline CT studies included & 67 \\
Patients with ZT & 56.1 \\
Mean age (years) & 45 \\
Males & 31 \\
Females & $60(89 \%)$ \\
Right & $49(73 \%)$ \\
Left & $42.1 \%$ \\
Percentage of patients with nodular appearance of ZT
\end{tabular}

extend into the tracheoesophageal groove or retroesophageal region. ${ }^{9}$

\section{Materials and Methods}

\section{Patient Selection}

Approval for this study was obtained from our institutional research ethics board. Informed consent was waived for this retrospective study. All neuroradiologists involved in image analysis in this study were certified in diagnostic radiology by the ABR, had completed a 2-year neuroradiology fellowship, and were eligible for, or certified with, an ABR Neuroradiology Certificate of Advanced Qualification. All CT studies performed per our neck protocol were identified on our PACS. We identified 145 sequential patients from January 2010 to July 2010. The CTs of the neck were performed on 40-, 64-, or 128section CT scanners (Siemens Definition AS Dual Source 64 section, Siemens Definition AS Plus 128 section, and Siemens Definition AS 40 section; Toshiba Aquilion One 320 section and Toshiba Aquilion

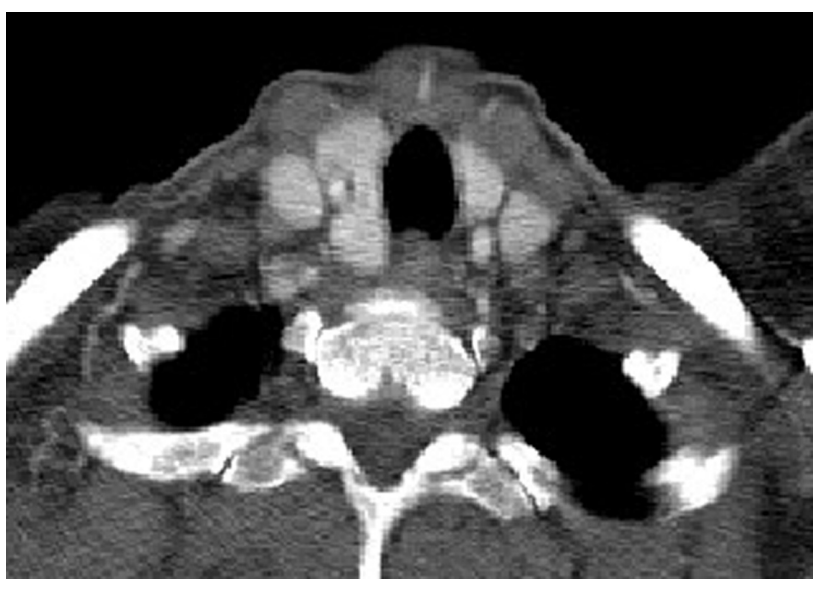

Fig 4. Patient $A$, with known ovarian carcinoma recurrence in the abdomen, presented with a few weeks' history of voice hoarseness and was noted to have a nodular area posterior to the right thyroid lobe. There was clinical concern for a metastasis or exophytic thyroid nodule.

64 section) with 3-mm section thickness axial imaging with coronal reformations. All studies were performed with contrast from the thoracic outlet to the skull base. Exclusion criteria included 1) patients with distorted thyroid anatomy due to previous surgery, large neck masses, or ectatic vessels, and 2) poor visualization of the thyroid secondary to artifacts. Overall, 145 sequential patients were reviewed and 95 patients were included for final analysis.

\section{Image Review}

We defined the part of the thyroid extending posterior to the tracheoesophageal groove on axial images as the ZT (Fig 2). The reason for 

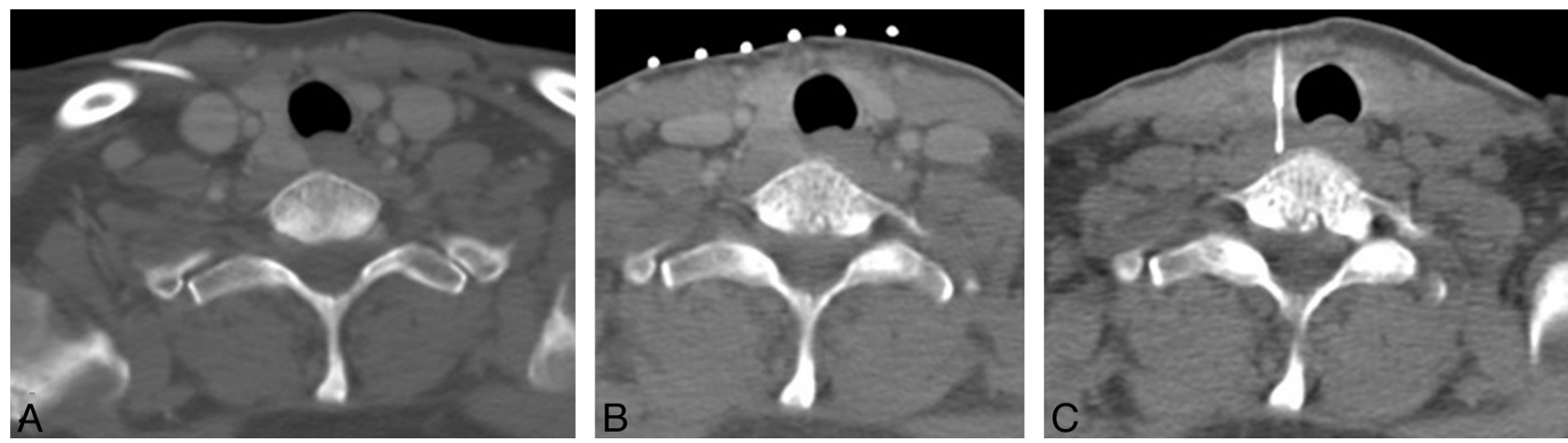

Fig 5. CT-guided biopsy of Patient A in 2010 with questionable thyroid lesion (A) was performed because a recent sonography-guided fine-needle aspiration was read as indeterminate by pathology and there was persistent clinical concern despite stability from a CT in 2006. The patient was placed on the CT table with neck extended, and a metallic grid marker was placed on the section, showing the epicenter of the questionable lesion. $B$, A metallic grid marker is in place for biopsy planning. There is shoulder streak artifact through the questionable lesion, which lowers the attenuation. C, CT-guided percutaneous core biopsy needle was placed in the epicenter. Pathology results were consistent with normal thyroid tissue, with no malignant cells.

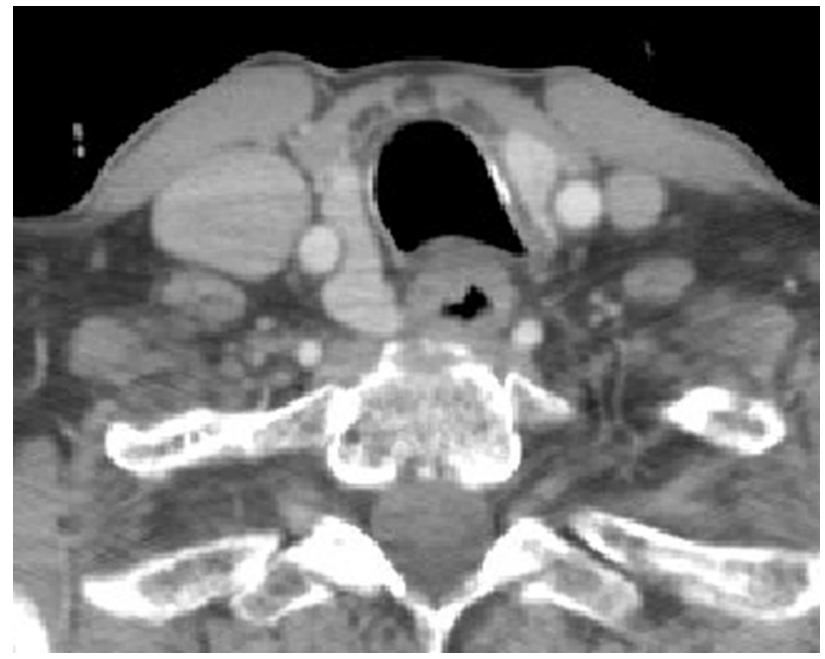

Fig 6. Patient A's pulmonary embolism chest CT from 2006 shows similar appearance of the questionable thyroid lesion.

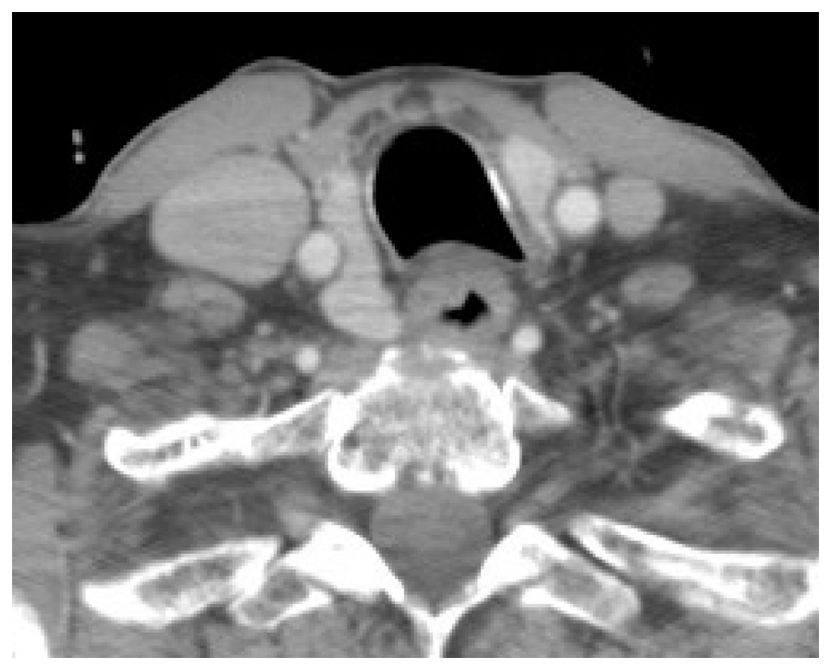

Fig 7. Patient B has similar appearance of the right thyroid lobe on a CT from December 2009, with a posterior extension on the right.

this criterion is that the recurrent laryngeal nerve has a fairly constant relationship to the ZT, which is usually posterior and lateral to the nerve; the recurrent laryngeal nerve is most often found in the tra-

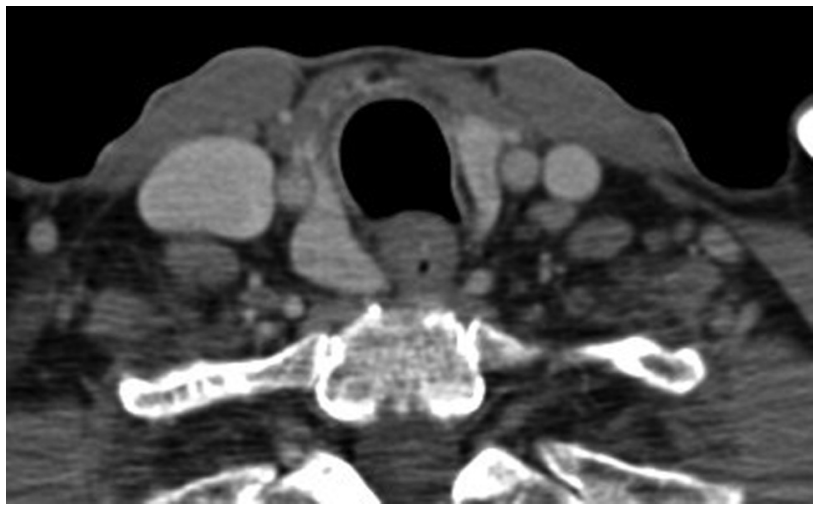

Fig 8. Patient B's thyroid appeared similar as far back as 2005, allowing for slight difference in the obliquity of the axial section.

cheoesophageal groove. The inferior thyroid artery, if identified, is usually closely associated with the ZT. The superior parathyroid gland is superior to ZT (Fig 1). There are variations in appearance of the ZT (Fig 3), ranging from absence of protuberance posterior to the tracheoesophageal groove to a nodular-like mass that can have a narrow neck or stalk from the posterior margin of the thyroid lobe and can even appear discontiguous from the thyroid gland.

\section{Results}

Of the 96 patients included in the study, 67 met CT imaging criterion for ZT (70\%) (Table). The mean age of these 67 patients was 56.1 years, with 45 males and 31 females. ZT was more often seen on the right: 60 tubercles were identified on the right $(89 \%)$ and 49 on the left $(73 \%)$. There were 40 patients with a nodular subtype of ZT on at least 1 side (Fig 3).

Patient A, our index case, is a 40 -year-old woman with recurrent ovarian cancer who presented with symptoms of hoarseness and was found to have a possible metastatic neck lesion. On imaging, the lesion appeared contiguous with the posterior aspect of the right thyroid lobe. On 1 neck CT, it had artifactually lowered attenuation due to streak artifact from the shoulders, but on another CT, it had similar attenuation and enhancement (Fig 4). The right common carotid artery was situated on the lateral aspect of the thyroid and the questionable metastatic lesion. The appearance was stable compared with a remote pulmonary embolism chest CT from 2006 

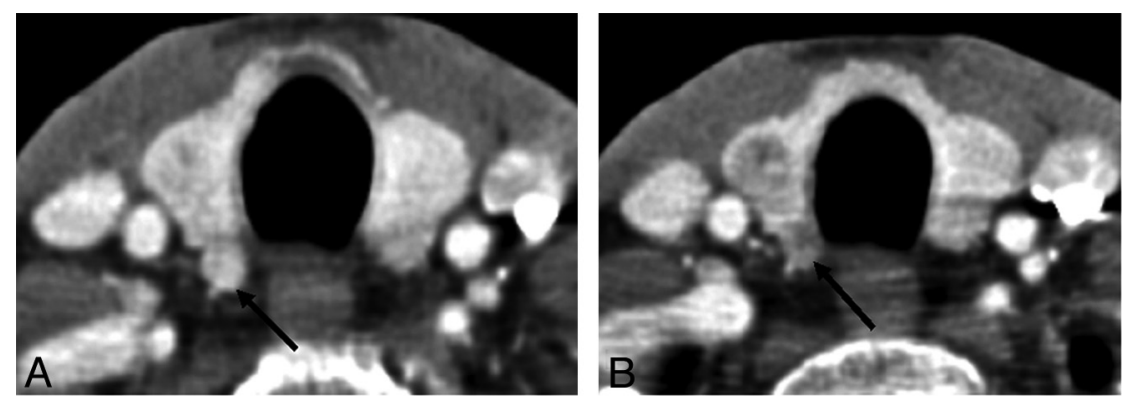

Fig 9. A, Thyroid gland with a nodular ZT at the posterior aspect of the right lobe (arrow). B, Follow-up CT study demonstrating development of a discrete hypoattenuated nodule in the right lobe and a hypoattenuated nodule in the previously identified ZT (arrow).
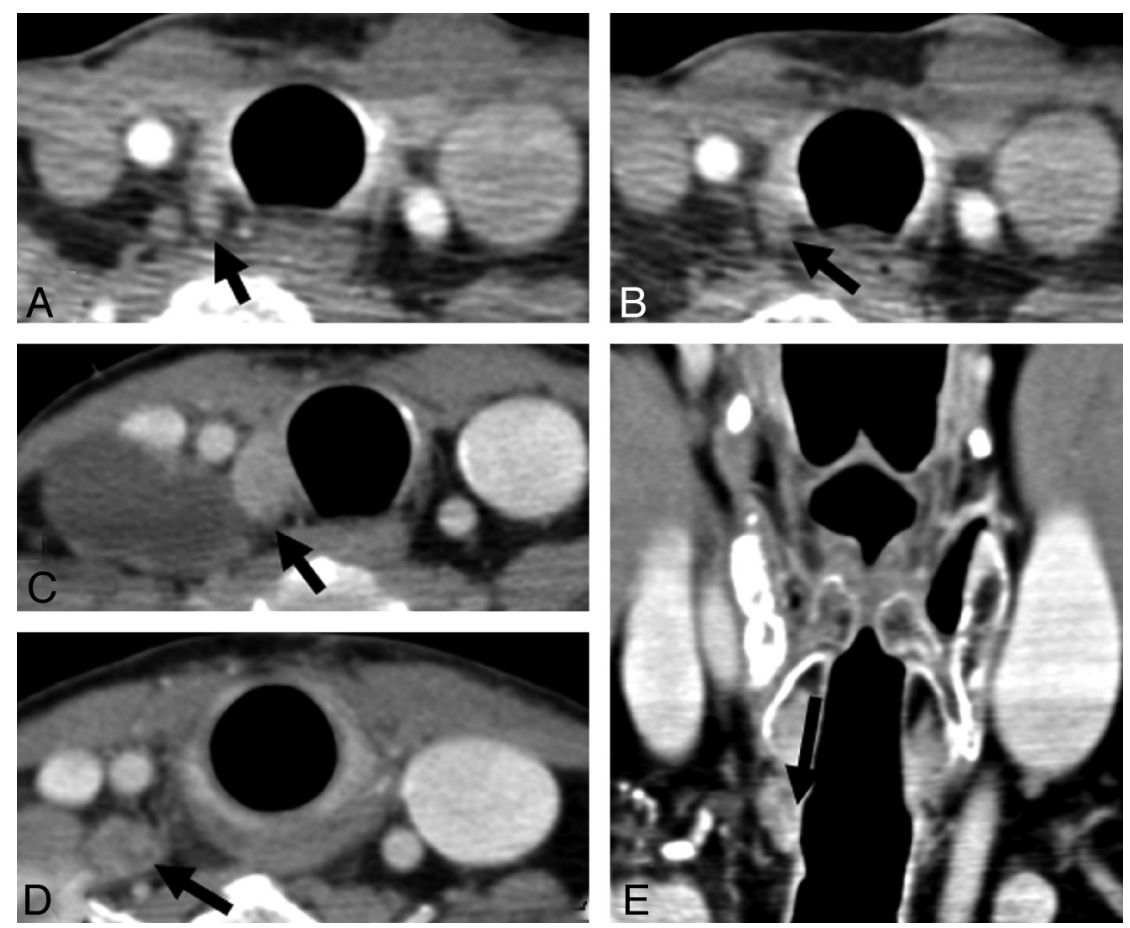

Fig 10. $A$ and $B$, CT images on a patient status post total thyroidectomy for thyroid cancer show a small enhancing lesion near the right tracheoesophageal groove (arrows), which likely represents a tubercle of Zuckerhandl. $C$ and $E$, Axial and coronal CT demonstrates enlargement of a paratracheal lesion after an interval of 6 months (arrows in $C$ and $E$ ); a nearby lymph node also showed enlargement (arrow in $D$ ) and proved to be recurrent disease.

(Fig 6), but due to the initial radiologic concern, a sonography-guided fine-needle aspiration of the nodular area was performed, with pathology read as indeterminate.

Due to ongoing clinical concern by the referring head and neck surgeon, a percutaneous core needle biopsy was performed at our institution (Fig 5), yielding normal thyroid tissue.

Patient B has similar appearance of a tubercle arising from the right thyroid lobe (Fig 7). This appearance was unchanged as far back as 2006 (Fig 8).

\section{Discussion}

The ZT is a normal surgical landmark of the thyroid gland with important anatomic and radiologic variation. It is characterized in the surgical literature as a protuberance on the posterior aspect of the gland that can be lifted to locate the recurrent laryngeal nerve in the tracheoesophageal groove. ${ }^{10-14}$ The fairly constant relationship of the tubercle with the superior parathyroid gland and recurrent laryngeal nerve helps in identifying these vital structures in surgery.
Sonography is widely considered the front-line of thyroid imaging, but the thyroid is typically seen on CT performed for other indications, and nodules or nodule-like regions of the thyroid are often visible. When the tubercle has a nodular shape, and particularly when the low neck is compromised by streak artifact from the shoulders, it can be mistaken as a mass or lymph node. The nodular subtype of ZT is common, occurring in more than a third of CT scans in our study, and is more commonly seen on the right. If it is not possible to determine on CT that a possible lesion is in fact a ZT, then sonography is generally the next step and biopsy can be avoided if the sonography is definitive.

In most cases of nodular ZT, there appears to be lateral indentation of the thyroid contour by the common carotid artery. This lateral indentation can appear, however, without the artery, and presumably is formed by a layer of pretracheal fascia, because the thyroid itself has no true capsule.

Although the ZT is composed of normal thyroid tissue, nodules may develop within the ZT just as in other normal thyroid tissue (Fig 9). The ZT is commonly left unresected if not involved 
by tumor to avoid harming the adjacent recurrent laryngeal nerve, and so it can be a future site for recurrence (Fig 10). Recognition of this aspect of thyroid anatomy can help avoid mistaking normal thyroid landmarks as nodules or lymph nodes.

\section{Conclusions}

ZT is a surgical landmark of the thyroid with important radiologic variations. Moreover, ZT occurs in a region where there is often artifact from the shoulders that can change the attenuation relative to the remainder of the thyroid and further contribute to its mimicry of a pathologic lesion. Recognition of this feature of thyroid anatomy allows one to avoid causing unnecessary concern on the part of patient and physician, avoid additional imaging, and avoid a biopsy and the attendant risks.

Disclosures: Srini Mukundan—UNRELATED: Consultancy: Toshiba America Medical Systems, ${ }^{*}$ Comments: Served on focus group; Grants/Grants Pending: Toshiba, ${ }^{*}$ Siemens; ${ }^{*}$ Payment for Lectures (including service on speakers bureaus): Toshiba, Comments: "Lunch and Learn" lecture at ASNR Annual Meeting in Vancouver (2009). ( ${ }^{*}$ Money paid to institution)

\section{References}

1. Mirilas P, Skandalakis JE. Zuckerkandl's tubercle: Hannibal ad Portas. Am Coll Surg 2003;196:5:796-801
2. Yalcin B, Tatar I, Ozan H. The Zuckerkandl tubercle and the recurrent laryngeal nerve. Am J Surg 2008;196:311-12

3. Costanzo M, Caruso LA, Veroux M, et al. The lobe of Zuckerkandl: an important sign of recurrent laryngeal nerve. Ann Ital Chir 2005;76:337-40, discussion 340-41

4. Musajo FG, Mangiante G, Ischia A, et al. Zuckerkandl tubercle of the thyroid gland (anatomo-surgical study: preliminary considerations). Chir Ital 1989; 41:129-36

5. Buck RT, Siddiqui AR. Thyroid abnormality secondary to tortuous carotid artery. Eur J Nucl Med 1986;12:51-52

6. Zuckerkandl E. Nebst Bermerkungen uber die Epithkorperchen des Menschen. Anat Hefte 1902;61:61

7. Gemsenjaeger E. Atlas of thyroid surgery: principles, practice, and clinical cases. Stuttgart, Germany: Thieme, 2005

8. Pelizzo MR, Toniato A, Gemo G. Zuckerkandl's tuberculum: an arrow pointing to the recurrent laryngeal nerve (constant anatomical landmark). J Am Coll Surg 1998;187:333-36

9. Ranade AV, Rai R, Pai MM, et al. Anatomical variations of the thyroid gland: possible surgical implications. Singapore Med J 2008;49:831-34

10. Delbridge L. Total thyroidectomy: the evolution of surgical technique. ANZ J Surg 2003;73:761-68

11. Page C, Cuvelier P, Biet A, et al. Thyroid ZT: anatomical and surgical experience from 79 thyroidectomies. J Laryngol Otol 2009;123:768-71.

12. Toniato A, Boschin IM. The Zuckerkandl tubercle. Am J Surg 2008;195:277

13. Gravante G, Delogu D, Rizzello A, et al. The Zuckerkandl tubercle. Am J Surg 2007;193:484-85

14. Gauger PG, Delbridge LW, Thompson NW, et al. Incidence and importance of the ZT in thyroid surgery. Eur J Surg 2001;167:249-54 